Mots. Les langages du politique

\title{
Une femme d'extrême droite dans les médias. Le cas de Marine Le Pen
}

Julie Boudillon

\section{(2) OpenEdition \\ Journals}

Édition électronique

URL : https://journals.openedition.org/mots/392

DOI : $10.4000 /$ mots.392

ISSN : 1960-6001

Éditeur

ENS Éditions

\section{Édition imprimée}

Date de publication : 1 juillet 2005

Pagination : 79-89

ISBN : 2-84788-080-1

ISSN : 0243-6450

Référence électronique

Julie Boudillon, « Une femme d'extrême droite dans les médias. Le cas de Marine Le Pen », Mots. Les langages du politique [En ligne], 78 | 2005, mis en ligne le 31 janvier 2008, consulté le 23 avril 2022. URL : http://journals.openedition.org/mots/392 ; DOI : https://doi.org/10.4000/mots.392 


\section{Une femme d'extrême droite dans les médias. Le cas de Marine Le Pen}

Apparue en plein «coup de tonnerre », pour reprendre une des expressions du lendemain du 21 avril 2002, Marine Le Pen est devenue en quelques mois une figure incontournable du Front national et sa présence soulève cette question : comment se dit et se montre la féminité à l'extrême droite? Le croisement des disciplines s'avère essentiel pour aborder cet objet « femme politique du Front national». En effet, l'analyse du discours nous fournit comme entrée théorique l'ethos, construction de l'image de soi dans le discours. Cependant, nous ne pouvons l'utiliser qu'en interrogeant la problématique du genre. Notre travail se propose en effet d'explorer l'hypothèse, déjà travaillée, de la plusvalue de la féminité en politique, autrement dit d'examiner le «retournement de stigmate » à l'épreuve du Front national, parti traditionnellement antiféministe. Ces résultats se fondent sur un corpus constitué d'articles de la presse nationale quotidienne et hebdomadaire, ainsi que de reportages télévisés et interventions de Marine Le Pen à des débats politiques ou dits "de société ". Si le corpus est hétérogène en termes de genre journalistique, c'est que l'analyse s'intéresse à une opinion publique généralisée. De fait, les résultats semblent indifférents aux variations de média ou de style.

La période concernée s'étend d'avril à décembre 2002, intervalle qui a vu la naissance politique autant que médiatique de cette nouvelle figure du Front national.

\section{L'ethos, de l'analyse du discours aux études de genre}

\section{Quelques précisions théoriques}

La notion d'ethos, ou image de soi dans le discours, nous vient de la Rhétorique d'Aristote qui la définit comme suit: "On persuade par le caractère, quand le discours est de nature à rendre l'orateur digne de foi, car les honnêtes gens nous inspirent une confiance plus grande [...]. Mais il faut que cette confiance soit l'effet du discours, non d'une prévention sur le caractère de

Université Paris 12, CEDITEC, julieb_fr@yahoo.fr 
l'orateur ${ }^{1}$. Largement reprise et redéfinie dans les sciences humaines, cette notion soulève des problèmes théoriques que nous ne pouvons intégralement évoquer ici. Il nous faut cependant tenter de synthétiser les précisions utiles dans le cadre de notre analyse. Établissons donc que:

- l'ethos relève de l'argumentation et, en tant qu'outil de persuasion, se doit d'être conforme à la doxa. Il mobilise pour cela des stéréotypes positifs en cours dans l'opinion publique;

- l'ethos se trouve en tension entre des schémas de pensée collectifs et une stratégie individuelle. L'image construite dans un discours correspond à des représentations collectives liées à des catégories sociales: discours de jeunes, discours de patrons, discours de femmes. Mais il existe une part de liberté individuelle; en cela, l'ethos rencontre une problématique centrale en analyse du discours : la tension entre les structures idéologiques et la liberté relative du sujet par rapport à ces contraintes. L'analyse du discours ne tente pas de déceler la sincérité de l'ethos, mais de repérer la représentation véhiculée et de cerner la stratégie du locuteur;

- il est primordial de considérer l'ethos comme une coconstruction : dans le cadre de notre analyse, il n'est pas le fait d'un seul locuteur, mais de sources énonciatives différentes, politiques et médiatiques. Ce qui nous intéresse, c'est le flux polyphonique produit sur et par Marine Le Pen, autrement dit son auto-/hétéro-représentation.

Ici, l'ethos n'est plus seulement un moyen de persuasion de l'orateur comme chez Aristote, mais devient, comme les sciences humaines le définissent également, une image définie en fonction de la doxa.

\section{Le retournement de stigmate}

Des observations menées sur des corpus récents révèlent que le stigmate de la féminité pouvait constituer un argument positif dans le discours politique. Nous considérons, dans la lignée des travaux menés sur le genre en politique, que la féminité constitue un stigmate dans la mesure où elle restreint les rôles des femmes dans l'espace public et les éloigne de la sphère politique, ou en tout cas leur en rend l'accession malaisée. Or, il semblerait que ce qui les tient éloignées peut parfois contribuer à légitimer leur présence en politique. Les stéréotypes féminins de la douceur, de la compassion, jusque-là propres au monde privé de la famille et du foyer (et qui se traduisait en politique par la désignation de certaines femmes à des postes à vocation humanitaire et sociale), sont désormais valorisés et constituent des arguments positifs en réaction à une vision dévalorisée de la politique.

1. Aristote, Rhétorique, édition de 1991, traduction de M. Dufour, Paris, Gallimard. 
«Mère de trois jeunes enfants, je veux rendre l'espoir à tous ceux qui souffrent », annonce Marine Le Pen sur la page d'accueil de son site Internet: avec cette relation de cause à effet induite par la syntaxe, la maternité tient lieu de ressource utile à la communauté. Ce qui cantonnait jusqu'à présent les femmes dans le rôle privé de mère au foyer devient atout: c'est dire que les compétences valorisées ne sont pas d'ordre politique mais plutôt privé; cette professionnalisation de l'intimité ou publicisation du privé devient d'ailleurs pratique courante en politique, chez les femmes comme chez les hommes.

\section{Ethos féminin positif au Front national}

Marine Le Pen n'échappe pas aux représentations classiques des femmes en politique, étudiées notamment par Mariette Sineau (Sineau, 1988; 2001) et Jane Freedman (Freedman, 1997). Assignées au monde privé de la famille et du foyer versus le monde public de la politique, les femmes restent cantonnées aux rôles de fille/épouse/mère. Ces traits sont quasi systématiques dans la construction de leur ethos, et constituent presque une grammaire de la représentation des femmes en politique. Nous n'en retiendrons, pour le présent article, qu'un seul: la filiation, notamment parce qu'elle est largement évoquée (par les médias) et revendiquée (par l'intéressée).

À de nombreuses reprises, l'entrée de Marine Le Pen en politique est expliquée par les médias ou par elle-même. En observant notre corpus, nous avons distingué deux justifications de sa carrière politique : celle de sa filiation en tant que telle, et celle des expériences douloureuses qu'engendra la vie politique de son père. Répertorions ces évocations avant d'en tirer des conclusions sur la construction de l'ethos.

\section{Filiation et vocation politique}

Héritage paternel revendiqué: Marine Le Pen n'échappe pas à un trait courant chez les femmes en politique. Cette ascendance légitimante est largement mise en récit dans son discours et celui des médias.

Le piège de la politique s'est en effet refermé sur elle il y a bien longtemps. Elle avoue volontiers sa passion pour la parole publique, le lien fusionnel avec sa famille [...]. Contre sa sœur [Marie-Caroline], sa mère, contre le monde entier, Marine a toujours pris le parti de ce père adoré. (Le Point, 24 mai 2002)

La passion de la politique s'était emparée d'elle. "Quand il a décidé de ne pas céder au chantage mais de virer Mégret, quel qu'en soit le prix, j'ai vu, confie-t-elle, l'incroyable force de mon père. II m'a appris à ne jamais baisser les bras. » (Le Figaro, $1^{\text {er }}$ juin 2002) 
Le discours de Marine Le Pen décrit l'héritage paternel comme un apprentissage de valeurs positives. En revanche, les commentaires journalistiques mobilisent le champ lexical de la passion, terme explicitement employé dans les deux extraits. Les syntagmes nominaux «piège/passion de la politique », ainsi que les verbes qu'ils régissent, filent la métaphore d'une prisonnière passionnée, prise dans les filets de la politique comme dans ceux d'un sentiment amoureux; on le voit, ces évocations puisent dans l'interdiscours culturel, littéraire mais aussi politique. L'image de la femme conduite par ses passions est d'usage courant: "Souvent, elle a eu la tentation de tout abandonner. Cette vie, son nom, la politique. Et puis, c'était plus fort qu'elle. » (Le Figaro, 10 juin 2002)

Qu'est-ce donc qui est plus fort qu'elle? "C'», instance non définie, non nommée, évoquée comme un fatum en marche contre lequel Marine Le Pen ne peut rien. La construction syntaxique de la phrase nominale agit comme un rythme entêtant: l'esthétisation de l'écriture journalistique contribue à la mise en récit d'une passion irréfléchie. L'engagement de Marine Le Pen relève donc moins d'un choix politique que d'une fatalité familiale.

\section{Par où le pathos s'engouffre: le discours de la souffrance}

Le 28 novembre 2002, Le Monde publie un article intitulé «Le Pen et filles», portrait de famille qui décrit les liens familiaux comme un embrigadement idéologique, consécutif au rejet du Front national par l'opinion publique.

Elles sont blondes. Elles sont sœurs. Elles sont trois. Elles connaissent depuis longtemps ce petit mouvement de recul qui saisit les autres dès l'instant où elles sont identifiées: les filles de Jean-Marie Le Pen. Marie-Caroline, Yann et Marine n'ont jamais pu y échapper [...]. Héritières d'un homme qui regretta longtemps de ne pas avoir eu de garçon pour perpétuer son nom et qui, aujourd'hui, assure tranquillement: «Mes filles ont toujours eu les mêmes opinions politiques que moi. C'est assez normal, elles aimaient leur père... » [...] « Pendant toute notre jeunesse, ce rejet nous a soudées comme des prisonniers dans une prison », dit Yann aujourd'hui.

On découvre alors l'histoire et la situation politique du Front national sous un jour nouveau ; dès lors le pathos s'engouffre, peut-on dire, dans le discours, et ce, quelle que soit la source énonciative:

[Marine Le Pen :] Quand on est la fille Le Pen, on apprend très jeune à souffrir. Les profs nous racontent exprès la guerre d'Algérie sous son jour le plus noir, les amis qui ne vous invitent pas aux anniversaires, les petits copains qui vous quittent parce qu'ils n'assument pas de sortir avec la fille Le Pen, sa mère à poil dans PlayBoy... (Le Figaro, 10 juin 2002)

Le $1^{\text {er }}$ novembre, vingt kilos d'explosifs détruisent l'immeuble où vivaient les Le Pen. Le visage grave, Marine se remémore cet instant. Elle se rappelle du «petit Guillaume», éjecté par la déflagration. Le bébé du voisin qui était dans son lit est tombé du cinquième étage et a atterri dans la rue, miraculeusement protégé par 
son matelas [...]. Des humiliations et de nombreuses réflexions ont été le «terreau de ma conscience politique», déclare-t-elle [...]. À 16 ans, elle tombe sur la une de Libération: "Torturés par Le Pen», le 12 février 1985. Alors, difficile d'avoir des copains quand on porte un tel nom. "Certains considérant que ce n'était pas très avantageux pour eux d'être l'ami ou le petit ami de la fille de Jean-Marie Le Pen.». (VSD, 24 octobre 2002)

Venue à la vie consciente en 1975 avec l'attentat à la bombe de la rue Poirier au domicile familial - elle avait 8 ans -, elle a vécu son adolescence avec le divorce fracassant de ses parents - fin 1984 - qui exposèrent leur haine dans les pages du magazine Lui. Elle a grandi au milieu des mélodrames cinglants. (Le Point, 22 novembre 2002)

La mise en scène dramatisée de l'adolescence troublée de Marine Le Pen puise dans l'interdiscours les motifs des grandes sagas familiales qui font les beaux jours de la presse people: famille célèbre, enfants maudits, évènements dramatiques...

Vraisemblablement, on bascule dans un autre genre discursif que le discours politique, bien qu'il s'agisse du reflet de la propension actuelle à faire accéder le discours de l'intime à l'espace public. Cette tendance est d'autant plus forte que le dispositif télévisuel est différent des débats politiques classiques; ainsi lorsque Marine Le Pen participe à l'émission de Bernard Tapie, elle ne parle de rien d'autre que de cette souffrance originelle. Extraits :

B. T. : Et au moment où il y a eu la fâcherie entre votre père et votre mère, [...] le fait d'avoir fait un choix sans ambigüité, total, complet, pour votre père, vous a posé un problème ou non?

M. L. P. : Non.

B. T. : Aucun?

M. L. P.: Non, aucun. [...]

B. T. : C'est rare qu'une enfant...

M. L. P. : Mais c'est très triste!

B. T. : Quel âge vous aviez?

M. L. P. : J'avais 16 ans.

B. T. : Voilà ; et à 16 ans, on n'a pas envie, plus, de secourir son père, sa mère, que d'aller...

M. L. P. : Monsieur Tapie, à 16 ans on a envie d'avoir son papa et sa maman, j'crois que tous les enfants du monde sont comme ça! [...]

Bien que faisant partie d'une émission dite «de société », cette interview correspond avant tout à une tendance de la télévision observée depuis une dizaine d'années, celle des reality shows, étudiée notamment par Dominique Mehl (Mehl, 1994). Insistant sur l'hétérogénéité de ces programmes, elle leur attribue cependant un point commun - la participation de personnes subissant la «solitude sociale»:

Ainsi, les candidats à la réparation télévisuelle, bien qu'appartenant à des milieux sociaux intégrés, bien que maîtrisant les codes culturels des couches cultivées [...], 


\section{MARINe Le Pen}

souffrent d'un chaînon manquant, d'un défaut d'insertion relationnelle. Ni exclus du travail, ni marginaux du logement, ils sont des exclus du sentiment et des marginaux du lien social. (p. 106)

Or, ce dont témoigne Marine Le Pen relève de la même carence :

[Marine Le Pen] : Ce que j’ai évidemment subi parce que je n'avais pas ma liberté de gestion de ma propre vie quand j'étais enfant, c'est son engagement politique tout entier, [...] les réactions qu'a eu le, une partie d'ailleurs du public tout à l'heure [= sifflements et huées au moment où Bernard Tapie présente Marine Le Pen], vous savez je les ai vécues beaucoup plus jeune. Et je crois que si je peux admettre aujourd'hui même si je trouve qu'elles sont déplacées parce que je trouve qu’il y a un certain respect à avoir vis-à-vis de qui que ce soit, je les ai très mal vécues quand j'étais plus jeune, parce que quand ça s'adresse, ces lazzis, ces quolibets, comme dit, comme il disait, quand ça s'adresse à une enfant, je trouve que c'est inadmissible, voilà ce que j’ai vécu moi.

Comme les participants des reality shows, Marine Le Pen se décrit souffrant d'exclusion. Selon D. Mehl, le passage à ce genre d'émission est motivé par la demande d'une réparation et constitue une «bouée de sauvetage pour des exclus de la richesse affective » (p. 105).

L'évocation des souvenirs d'enfance de la locutrice, vécus et racontés sur le mode du traumatisme, s’inscrit dans le discours ambiant télévisuel destiné, selon D. Mehl, à « légitimer la parole souffrante» (p. 104). Peut-on y voir, de la part de la locutrice politique, une instrumentalisation de son propre passé, allié à une tendance générale du discours télévisuel?

\section{Une nouvelle pasionaria}

Au regard de ces observations, Marine Le Pen bénéficie en partie du même traitement médiatique que d'autres femmes politiques: entrée en politique par passion plutôt que par ambition, impliquée personnellement dans son combat, elle correspond à la figure de la pasionaria, qui implique que l'action politique féminine ne peut être qu'irréfléchie.

Cette image, à la fois traditionnelle (en tant que femme politique, elle est une pasionaria) et inédite (ses souvenirs-traumatismes expliqués et relayés par les médias), a deux effets sur l'ethos de Marine Le Pen:

- d'une part, elle légitime sa présence en politique : son engagement n'est pas dû au seul fait d'être la « fille de », mais également d'avoir souffert, dans sa jeunesse, de la mauvaise image de son père. Ayant eu sa part de souffrance, Marine Le Pen peut maintenant s'engager en politique : les expériences d'ordre privé, qui relèvent essentiellement du ressenti, feraient-elles figure d'arguments légitimants, plus que la filiation ou même la pratique? Cette légitimation, inédite nous semble-t-il, redéfinit-elle la chose politique? 
- d'autre part, et c'est le deuxième aspect de l'image de Marine Le Pen, on assiste à la construction d'un ethos inédit au Front national. L'image de JeanMarie Le Pen dénonçant un complot politico-médiatique créait jusqu'à présent un malaise chez les journalistes, qui oscillaient entre l'accusation de paranoïa et la remise en question de leurs propres méthodes. Aujourd'hui, l'énonciateur a changé: il ne s'agit plus d'un homme politique à l'ethos très marqué, mais d'une petite fille traumatisée par une explosion, puis d'une adolescente au comportement rebelle, et enfin d'une femme politique qui assume tous ses choix. La souffrance ressentie, puis décrite par la locutrice comme par les médias, qui, pour la plupart, la mettent en récit dans un souci de dramatisation, peut rendre le personnage touchant; on assiste à un véritable retournement des arguments jusqu'ici utilisés contre le Front national: «On dit que c'est nous qui sommes violents. Moi, ça ne m'atteint pas. Je suis une vieille femme en politique.»

Cet ethos peut paraitre en contradiction avec l'image jusqu'ici dominante au Front national: l'évocation d'une enfance douloureuse a peu de rapport avec l'image de Jean-Marie Le Pen, issu de l'extrême droite traditionnelle, à la carrure imposante, au passé militaire.

\section{L'ethos à rebours de la féminité}

L'«expression de soi », positive dans le discours politique, n'est cependant pas la seule composante de l'image de Marine Le Pen. L'héritage dont elle se réclame n'est pas exempt de conséquences dans la construction de son ethos: dans la doxa journalistique, un certain nombre de représentations négatives circulent autour du Front national : violence, vulgarité, généralement associées au genre masculin. Représentations qui prennent corps, au sens propre, chez Marine Le Pen.

\section{La virilité en héritage}

La ressemblance physique au père est souvent, sinon systématiquement, soulignée; réelle ou non, cette ressemblance n'est pas flatteuse par rapport à nos normes de la féminité, et tend aussi à stigmatiser un comportement viril.

Des trois filles du «menhir», c'est Marine qui ressemble le plus à son père. «Avec des cheveux », dit-elle. Trente-quatre ans, massive et rentre dedans [...]. De ce père provocateur, la petite dernière a hérité les idées - «à $300 \%$ » note sa sœur Yann, la plus discrète. Mais aussi le caractère rebelle, un peu vulgaire, et le tempérament turbulent. (L'Express, 17 octobre 2002)

La grande blonde à la voix rauque, les lèvres minces, l'œil vert vif, le visage parsemé de taches de rousseur, empoigne son portable dans un restaurant chic de SaintCloud. (Le Point, 22 novembre 2002) 
Toute cette description est fondée sur le principe du mélange des genres (masculin/féminin) : elle est «blonde» mais sa voix est «rauque»; elle se trouve dans un restaurant «chic », mais «empoigne» son portable. Attitude masculine dans un décor raffiné: Marine Le Pen semble oublier les normes de la féminité. Si Jane Freedman remarque que

le comportement de ces femmes [politiques] est présenté comme «déviant», en ce qu'elles traversent les frontières entre les domaines assignés aux deux sexes. L'image d'elles qui se présente dans le discours journalistique est l'image de femmes «masculinisées", des femmes qui adoptent des comportements masculins et qui sont dotés de qualités viriles (Freedman, 1997, p. 173),

nous pouvons risquer d'évoquer, dans le cas de notre analyse, au-delà d'une «masculinisation », une « lepénisation » (ou plutôt « jean-marie-lepénisation »!) des attitudes :

«Parfois, il faut envoyer les paras pour libérer la population des troupes d'occupation socialo-communistes », s'amuse l'ex-avocate, hâbleuse, entre deux lampées de vin blanc, dans un bar de militants FN, pavoisé aux couleurs du RC Lens. Ses partisans, attablés devant une mousse posée sur les tables de Formica, boivent des yeux la pasionaria nationaliste, et écoutent en silence ses coups de gueule contre «le terrorisme intellectuel ambiant». (Le Point, 24 mai 2002)

Significativement, la référence à la pasionaria est ici convoquée dans un portrait qui tend à décrire Marine Le Pen comme un prédicateur populaire, qui évolue dans un décor tout aussi populaire. Virilité et populisme sont d'ailleurs souvent associés dans les descriptions de Marine Le Pen :

Dans la bagarre, son verbe devient vite hargneux, et elle peut se révéler odieuse. «Une grande gueule, avec un rire de 6 sur l'échelle de Richter», décrit Yann [...]. C'est surtout une présence, imposante [...]. Avec son air batave et sa gouaille potache, Marine Le Pen est à l'aise avec le populo. (L'Express, 17 octobre 2002)

On est loin d'une féminité discrète et charmante. L'adjectif batave, nom des tribus d'outre-Rhin envahies par les Romains, plus tellement usuel, évoque la germanité. Bataves désigne également les habitants des Pays-Bas. L'intertexte ( " hargneux », " grande gueule », «présence imposante », " gouaille », etc.) tisse la connotation du stéréotype négatif de l'Allemande grossière, «hommasse», dont a pu se régaler la vulgate populaire française pendant des décennies.

La ressemblance physique qui accompagne une ressemblance comportementale, et donc éloignée de la féminité, s'accompagne du motif du clone: "Cette fois, il est question d'une fille, la benjamine, dont la stature, le verbe et l'appétit de vivre la font surnommer “le clone” »(Le Monde, 13 juillet 2002).

Figure symptomatique d'une menace, en raison des spéculations scientifiques et de la production artistique de type science-fiction, l'idée de clone peut également donner lieu à des jeux sur l'analogie des prénoms : le Canard enchaîné titre "Jean-Marine Le Pen»; "La plus Jean-Marine», commente le 
journaliste de Libération. Marine Le Pen devient alors créature hybride, mihomme mi-femme, voire mi-père mi-fille.

Cela étant dit, nous ne pouvons être sûre qu'en termes de stratégie de communication, cette image «virile» soit toujours dévalorisante pour l'intéressée. Le motif du clone, comme celui de la prédicatrice populaire, est sans doute négativement connoté dans la presse nationale parisienne; mais il semblerait que Marine Le Pen, qui d'ailleurs se réclame parfois de la ressemblance physique avec son père, s'approprie l'ethos de son père, marque de fabrique garante en partie de son succès électoral. En réalité, face à l'électorat du Front national, elle construit sa légitimité en jouant sur cet aspect viril, constitutif de l'ethos populiste des leaders d'extrême droite.

\section{La racoleuse}

Parfois, la représentation de Marine Le Pen s'écarte tellement des stéréotypes positifs de la féminité politique qu'elle est associée à la figure de la prostituée racoleuse. Le terme peut être employé explicitement, comme dans ce billet d'humeur de Libération:

Bien sûr, «la blonde », comme on dit dans les bistrots où elle racole (1) l'électeur, a
assez appris de ses études d'avocate pour ne pas s'aventurer sur les terrains maré-
cageux d'un « humour mal venu [qui] s'exerce aussi contre les juifs dans des calem-
bours plus que douteux ». (Cour d'appel de Chambéry, 4 novembre 1992).

(1) $\searrow$ 1. Anciennt. Enrôler par force ou par ruse, en violation déguisée du principe de l'engagement volontaire. $\backslash 2$. (Av. 1794) Mod. Attirer, recruter par la parole, par des moyens publicitaires. (Marine Le Pen étant la procédurière fondatrice du service juridique de FN, nous citons ici le dictionnaire Robert pour prévenir la mise en cause de notre «bonne foi »). (Libération, 14 juin 2002)

Le journaliste n'évoque qu'un des trois sens du verbe, souvent utilisé pour dénigrer les pratiques politiques: l'utilisation de ce terme parait donc légitime. Mais le jeu sur la polysémie est travaillé : en éludant la dernière définition du terme, il se dédouane de tout sous-entendu et pourtant le formule à travers l'intertexte : "la "blonde" », le registre familier du terme bistrot sémantisent cette signification. Le décor planté est celui de la prostitution populaire, celle des bars et des trottoirs.

La même connotation apparait dans un article au titre évocateur, « Marine Le Pen drague les métallos »:

La caravane du Front national s'est installée dans le Pas-de-Calais minier. Marine Le Pen, jupe moulante sur talons hauts, attire le chaland derrière cette nouvelle vitrine. Elle harangue les journalistes, 40 pour elle toute seule, le dernier jeudi de mai, sur le marché d'Harnes. (Marianne, 10 juin 2002)

Le décor est sans équivoque: une caravane, une femme habillée en «jupe moulante sur talons hauts »... "Attirant» et " haranguant», Marine Le Pen est 
assimilée à une prostituée du bois de Boulogne. Cette image est confortée par la formule « 40 pour elle toute seule », qui transforme les journalistes en clients.

Si Marine Le Pen se voit affublée, comme d'autres femmes politiques, de qualités viriles, elle est cependant l'objet de descriptions insultantes qui en font un cas à part dans la classe politique féminine.

Au regard de ces analyses, nous pouvons conclure que Marine Le Pen mobilise un ethos inédit, ou plutôt un usage inédit de la féminité dans la construction de son ethos.

La souffrance évoquée par Marine Le Pen agit d'abord comme une véritable valeur ajoutée en politique. Cet usage est rendu possible, d'une part, par l'appartenance au genre féminin, réputé enclin à mobiliser cette « expression de soi ", et de l'autre, par le discours médiatique ambiant - ce que D. Mehl appelle le "pacte compassionnel», propre aux émissions de reality show, concerne également tout un aspect récent de la chose politique :

Ces émissions [de reality show] sont tout à fait congruentes avec la politique du moment. Le protocole compassionnel, mis en scène par les médias, est parent de l'action humanitaire. Il fonctionne sur le même registre: susciter l'émotion par la monstration de la misère, en faire partager imaginairement l'expérience, faire naitre la solidarité par identification. (Mehl, 1994, p. 116-117).

Ce qui nous semble également inédit, ce sont les références explicites à la prostitution. Les postures ouvertement dégradantes dans lesquelles est décrite Marine Le Pen sont loin d'être pratique courante dans la représentation des femmes politiques telle qu'elle se pratique aujourd'hui : ici, on n'est plus dans la présentation de soi contrôlée par l'intéressée mais dans la construction médiatique par une presse qui lui est hostile.

Mais d'une façon ou d'une autre, ce sont bel et bien la féminité de Marine Le Pen et l'usage des stéréotypes liés aux genres qui définissent les divers aspects de cet ethos: la féminité positive de la pasionaria souffrante légitime, d'une autre manière (une nouvelle manière), sa présence en politique.

Quant à la féminité en négatif, «la féminité masculine», vulgaire, elle lui permet de s'approprier des aspects virils, certes dévalorisés dans certains milieux, mais sans doute bien perçus par toute une frange de la population, celle des électeurs du Front national : cette femme «qui en a » a la poigne suffisante pour reprendre le flambeau Le Pen.

Ce jeu sur les genres, tantôt légitimant, tantôt délégitimant, nous montre à quel point ce qui relève de la stratégie de communication et des représentations médiatiques est inextricable dans la construction de l'ethos. 


\section{Références}

AMOSSY R. éd., 1999, Images de soi dans le discours. La construction de l'ethos, Paris, Delachaux et Niestlé.

ARISTOTE, édition 1991, Rhétorique, Paris, Gallimard, traduit par Médéric Dufour.

BERTINI M.-J., 1991, Femmes, le pouvoir impossible, Paris, Pauvert.

BONNAFOUS S., 1997, "Jean-Marie Le Pen et les médias», La question médiatique, Paris, Seli Arslan, p. 101-113.

— 2002, "La question de l'ethos et du genre en communication politique », Actes du premier colloque franco-mexicain en information et communication, publication en ligne: http://www.cerimes.fr/colloquefrancomexicain/et sur un CD-Rom édité par la SFSIC (Société française des sciences de l'information et de la communication).

— 2003, "“Femme politique" : une question de genre? », Réseaux, n 120, p. 121-145.

DULONG D., MATONTI F., 2003, "L'indépassable féminité. La mise en récit des femmes en campagne», J. Lagroye, P. Lehingue, F. Sawicki éd., Mobilisations électorales. À propos des élections municipales de 2001, Paris, PUF, CURAPP/CRAPS.

FREEDMAN J., 1997, Femmes politiques: mythes et symboles, Paris, L'Harmattan.

MAINGUENEAU D., 2000, "Lecture, incorporation et monde éthique», Études de linguistique appliquée, $\mathrm{n}^{0} 119$, p. 265-275.

— 2002, "Problèmes d'ethos », Pratiques, nº 113-114, p. 57-67.

MEHL D., 1994, "La télévision compassionnelle », Réseaux, Paris, nº63, p. 103-121.

PASTEUR P., 2004, "Le populisme: un genre masculin», C. Bard éd., Le genre des territoires, Presses de l'université d'Angers, p. 149-164.

SINEAU M., 1988, Des femmes en politique, Paris, Economica.

— 2001, Profession: femme politique, Paris, Presses de Sciences Po.

VENNER F., 1999, "L'extrême droite et les femmes ", C. Bard éd., Un siècle d'antiféminisme, Paris, Fayard, p. 417-430. 